\title{
Prognostic Value of Systemic Immune-Inflammation Index in Patients with Testicular Cancer: A Retrospective Case- Control Study
}

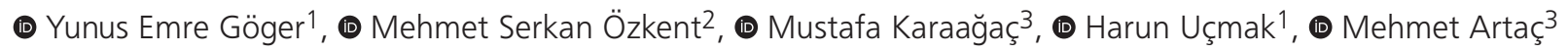 \\ ${ }^{1}$ Necmettin Erbakan University Meram Faculty of Medicine, Department of Urology, Konya, Turkey \\ 2Konya City Hospital, Clinic of Urology, Konya, Turkey \\ ${ }^{3}$ Necmettin Erbakan University Meram Faculty of Medicine, Department of Medical Oncology, Konya, Turkey
}

\begin{abstract}
Objective: The primary aim of this study was to evaluate the correlation between the systemic immune-inflammation index (SII) and clinicopathological outcomes of patients with testicular cancer (TCa). The secondary aim was to evaluate the relation of SII with overall survival (OS).

Materials and Methods: A total of 244 patients were included in the study. Patients were divided into the testicular tumor (group 1, n=184) and control group (group 2, n=60). Preoperative complete blood count, tumor markers, and imaging tests of the patients in group 1 were recorded. A subgroup analysis was performed according to the clinical stage, pathological stage, tumor type, and tumor size. Then, the effectiveness of TCa on SII was evaluated among the groups. Results: A significant difference was observed between the SII, neutrophil, and neutrophil-to-lymphocyte ratios between groups 1 and 2. The median SII was 719.92 in group 1 and 510.93 in group $2(p<0.001)$. In the subgroup analysis, the median Sll value was higher in patients with advanced disease stage and metastasis $(p<0.001)$. In the receiver operating characteristics curve analysis, the area under the curve was 0.784 , and the SII cut-off point was 719 , with a sensitivity of $81 \%$ and specificity of $65.4 \%$. The median follow-up time was 55 (interquartile range, 8-132) months. Ten patients died of TCa. In the multivariable analysis, SII (7.6-fold increase; $p=0.005$ ) and presence of metastasis (4.3-fold increase; $p=0.001$ ) were independent predictors of OS.

Conclusion: SII can be an important marker in the diagnosis and follow-up of TCa. However, SII needs to be evaluated using larger data, especially in the risk assessment in $\mathrm{TCa}$.
\end{abstract}

Keywords: Systemic immune-inflammation index, testicular cancer, cancer prognosis, overall survival

\section{Introduction}

In western countries, 3-10 in 100,000 men are diagnosed with testicular cancer (TCa) annually, representing $1 \%$ of all male neoplasms and $5 \%$ of all urological tumors $(1,2)$. The recurrence rate of $\mathrm{TCa}$ has been steadily increasing in recent decades, especially in developing countries (3). It is the most common solid tumor in men aged 20-34 years with a globally rising tendency (4). TCa is divided into two main subcategories, namely, seminoma and non-seminomatous germ cell tumor (NSGCT) that makes up $95 \%$ of all malignant tumors in the testes (5). According to a population-based patient series within developed countries, at the initial stage of diagnosis, stage I TCa is diagnosed in $75 \%-80 \%$ of patients with seminoma and in
$55 \%-64 \%$ of patients with NSGCT $(2,6)$. TCa survival outcomes are quite high with $95 \%$ of the patients attaining 5 -year cancerspecific survival (CSS) mainly due to early clinical staging of the tumor grade at TCa diagnosis. Only $10 \%$ of TCa cases present with metastatic disease, lowering the 5-year CSS to 73\% (4).

Inflammation has an important function in the biology and etiology of versatile tumors and is thought to be a characteristic of cancer (7). Several systemic inflammation markers (e.g., leukocytes, neutrophils, and thrombocytes) can be evaluated with simple and routine blood tests. Compared with platelet (Plt)-, neutrophil-, or lymphocyte-based tools, systemic immune-inflammation index (SII) emerges as a more powerful tool in cancer diagnosis and follow-up as it combines three independent prognostic factors $(8,9)$.

Cite this article as: Göger YE, Özkent MS, Karaağaç M, Uçmak H, Artaç M. Prognostic Value of Systemic Immune-Inflammation Index in Patients with Testicular Cancer: A Retrospective Case-Control Study. Bull Urooncol 2021;20(4):252-257

Address for Correspondence: Yunus Emre Göger, Necmettin Erbakan University Meram Faculty of Medicine, Department of Urology, Konya, Turkey Phone: +90 5334150753 E-mail: dr_yegoger@yahoo.com ORCID-ID: orcid.org/0000-0002-4480-9093 
Systemic inflammation markers and their ratios in particular have been verified for their prognostic values in malignancies such as genitourinary cancers like urothelial cancer, kidney cancer, and prostate cancer $(9,10,11,12)$. Compared with PIt-, neutrophil-, or lymphocyte-based tools, SIl emerges as a more powerful tool in cancer diagnosis and follow-up as it combines three independent prognostic factors (8). A high SII activity is considered a poor prognosis criterion, such as cancer progression, metastasis, and low overall survival (OS) $(13,14,15,16)$. However, studies on SII related to TCa are limited.

With the above background, the primary aim of this study was to evaluate the correlation between SII and clinicopathological outcomes, and the secondary aim was to evaluate the relation of SII with OS.

\section{Materials and Methods}

In this study, records of patients with TCa followed up at the urology and oncology clinics of Necmettin Erbakan University Meram Medical Faculty and at the Urology Clinic of Konya Training and Research Hospital between January 2008 and December 2020 were evaluated retrospectively. Cases with extragonadal GCT, missing information about systemic inflammatory markers, or incomplete follow-up information were excluded from the study.

Patients were divided into the testicular tumor group (group 1) and the control group (group 2). Group 1 consisted of patients with TCa, while group 2 was composed of patients without testicular tumors who presented to the hospital with different complaints, such as varicocele and hydrocele, during the same period.

Before radical orchiectomy, patients' age, complete blood count [hemoglobin $(\mathrm{Hb})$, neutrophils, Plt, mean platelet volume (MPV), lymphocytes, etc.], alpha-fetoprotein (AFP), beta-human chorionic gonadotropin (HCG), and lactate dehydrogenase values were recorded. Clinical staging was performed based on abdominal and thoracic computed tomography before treatment. Patients were classified according to age, TNM stage, and International Germ Cell Consensus Classification (IGCCCG) risk groups. Pathology results were recorded. According to patients' clinical and pathological stages, treatment and followup protocols were arranged according to the The European Association of Urology guideline. In addition, Hb, MPV, Plt, neutrophil-to-lymphocyte ratio (NLR), and SII (NxP/L) were calculated.

The recorded values were compared between the two groups. The relationship between SII value and pathological and clinical stages of TCa was evaluated, and the correlation between prognosis and SII value was also examined.

All procedures were performed in accordance with the ethical standards of the institutional and/or national research committee and with the 1964 Helsinki Declaration and its later amendments or comparable ethical standards. Written informed consent was obtained from all patients. The study was approved by the Meram Medical Faculty Ethics Committee of Necmettin Erbakan University (protocol no: 2021/2980).

\section{Statistical Analyses}

Non-parametric tests were used to analyze parameters that deviated from the normal distribution. Parameters with normal distribution were analyzed by parametric tests. Variables with continuous normality distribution were expressed as mean \pm standard deviation. Categorical variables were presented as percentage, and variables without normal distribution were expressed as median and interquartile ranges (IQR). The MannWhitney $U$ test or independent t-test was used to evaluate statistical differences between groups. The chi-square test was also used to analyze categorical variables.

Kaplan-Meier analysis was conducted to estimate OS using product-limit method, and log-rank test comparisons were performed subsequently. To define differences in Sll-based prognoses, a multivariable analysis was conducted using Cox proportional hazards model. The areas under the receiver operating characteristic curves (ROC) of SII were used to predict TCa.

Statistical Package for Social Sciences version 23.0 (IBM Corp., Armonk, NY, USA) was used to analyze data. A confidence interval $(\mathrm{Cl})$ of $95 \%$ and a $p$-value of $<0.05$ were considered for the threshold level of significance. All reported $p$-values were two-sided.

\section{Results}

A total of 244 participants were enrolled in this study, of which 184 (75.4\%) were classified in group 1 (TCa group) and 60 (24.6\%) in group 2 (control group). The mean age of all patients was $37.72 \pm 9.9$ (19-66) years, and those in groups 1 and 2

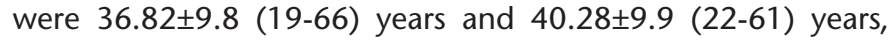
respectively. The median SII values were 719.92 (IQR: 225.732802.5) in group 1 and 510.93 (IQR: 235.24-1436.94) in group 2, in favor of group 1 (Mann-Whitney $U=1309 ; Z=-4.001$; $\mathrm{p}<0.001)$. The mean values of $\mathrm{Hb}$, Plt, lymphocytes, neutrophil, and MPV were 15.27 $\pm 1.7,279.68 \pm 62.3,2.17 \pm 0.6,5.96 \pm 2.1$, and $8.54 \pm 1.8$, respectively. The mean neutrophil, mean NLR, and median SII values were higher in group 1 than in group 2 $(p<0.001 ; p=0.002 ; p=0.001$, respectively) (Table 1$)$.

In this study, 78 (42.4\%) patients had NSGCT and 106 (57.6\%) had seminoma GCT. In addition, 123 (66.8\%) patients had stage I cancer, and 61 patients (33.2\%) had stage II and over. In the pathological evaluation, lymphovascular invasion (LVI) was present in 109 (59.2\%) patients (Table 2).

The mean tumor size was $4.55 \pm 2.1 \mathrm{~cm}(1-13 \mathrm{~cm})$. The median SIl value was 911.50 (IQR: $225.73-2802.5$ ) in tumors $\leq 4 \mathrm{~cm}$ in size. However, the SIl value was 827.57 (IQR: 355.93-2402.28) in tumors $>4 \mathrm{~cm}$ in size. No difference was found between the groups in terms of tumor size (Mann-Whitney $U=1124 ; Z$ -1.027; $p=0.235$ ) (Table 2). The median AFP and beta-HCG values were 2783 (IQR: 401-563495) and 5.38 (IQR: 0-265000), respectively.

The median SII value of 138 patients (75\%) with pathological T1-2 stage was 815.97 (IQR: 225.73-2201.4) and that of 46 patients (25\%) with pathological >T2 stage was 1631.71 (IQR: 513.6-2802.5). 
Regarding clinical tumor stage, the median SII value was 683.33 (IQR 225.73-2512.18) for clinical I stage tumors and 1036.00 (IQR 387.88-2802.50) for clinical stage II and over. The SII value was higher in advanced-stage tumors (Mann-Whitney $U=713$; $\mathrm{Z}=-3.652 ; \mathrm{p}<0.001)$.

In total, 32 (17.4\%) patients had metastasis: lung metastasis, 20 patients; liver and lung metastasis, 6 patients; bone metastasis, 5 patients; brain metastasis, 1 patient. The SII value was the highest in patients with metastasis than in those without it. The median SII value was 1204 (IQR 506.64-2802.50), and it was higher in patients with metastasis than in those without metastasis (Mann-Whitney $U=1518 ; Z=-5803 ; p<0.001$ ).

The SII value did not affect the LVI (Mann-Whitney $U=1544$; $\mathrm{Z}=-0.049 ; \mathrm{p}=0.961$ ). The median SII value was 715.81 (IQR 225.73-2310.8) in tumors without LVI and 810.06 (IQR 241.432802.50) in tumors with LVI (Table 2).

\begin{tabular}{|l|l|l|l|}
\hline \multicolumn{4}{|l|}{ Table 1. Demographic findings of the patients } \\
\hline & Group 1 & Group 2 & p-value \\
\hline Patients (n) & $184(75.4 \%)$ & $60(24.6 \%)$ & \\
\hline Mean age (years) & $36.82 \pm 9.8$ & $40.28 \pm 9.9$ & 0.062 \\
\hline Hemoglobin value & $15.27 \pm 1.7$ & $15.55 \pm 1.2$ & 0.172 \\
\hline Mean platelet level & $279.68 \pm 62.3$ & $272.45 \pm 57.3$ & 0.810 \\
\hline Mean neutrophil level & $5.96 \pm 2.1$ & $4.71 \pm 1.7$ & $<0.001$ \\
\hline Mean reticulocyte level & $13.06 \pm 1.8$ & $12.81 \pm 0.8$ & 0.399 \\
\hline Mean monocyte value & $0.59 \pm 0.1$ & $0.56 \pm 0.2$ & 0.437 \\
\hline Mean MPV & $8.54 \pm 1.8$ & $9.93 \pm 0.7$ & $<0.001$ \\
\hline Mean NLR & $2.88 \pm 1.4$ & $2.16 \pm 0.9$ & 0.002 \\
\hline Median SII & 719.92 & 510.93 & 0.001 \\
\hline Median AFP value & 2783 & N/A & N/A \\
\hline Median beta-HCG value & 5.38 & N/A & N/A \\
\hline $\begin{array}{l}\text { LVI: Lymphovascular invasion, NLR: Neutrophil-to-lymphocyte ratio, SII: } \\
\text { Systemic immune-inflammation index, MPV: Mean platelet volume, AFP: } \\
\text { Alpha-fetoprotein, HCG: Human chorionic gonadotrophin, N/A: Not } \\
\text { available }\end{array}$
\end{tabular}

\begin{tabular}{|c|c|c|c|c|}
\hline Variables & & $\begin{array}{l}\text { Patient } \\
\text { number (n) } \\
(\%)\end{array}$ & $\begin{array}{l}\text { Median SII } \\
\text { value }\end{array}$ & $P$ value \\
\hline $\begin{array}{l}\text { Pathological } \\
\text { stage }\end{array}$ & $\begin{array}{l}\mathrm{T} 1-2 \\
\geq \mathrm{T} 2\end{array}$ & $\begin{array}{l}138(75) \\
46(25)\end{array}$ & $\begin{array}{l}815.97 \\
1631.71\end{array}$ & 0.027 \\
\hline Size & $\begin{array}{l}\leq 4 \mathrm{~cm} \\
>4 \mathrm{~cm}\end{array}$ & $\begin{array}{l}101(54.9) \\
83(45.1)\end{array}$ & $\begin{array}{l}911.50 \\
827.57\end{array}$ & 0.235 \\
\hline Pathology & $\begin{array}{l}\text { Seminoma } \\
\text { Non-seminoma }\end{array}$ & $\begin{array}{l}106(57.6) \\
78(42.4)\end{array}$ & $\begin{array}{l}752.34 \\
801.45\end{array}$ & 0.084 \\
\hline Stage & $\begin{array}{l}\text { Stage I } \\
\text { Stage } \geq \text { II }\end{array}$ & $\begin{array}{l}123(66.8) \\
61(33.2)\end{array}$ & $\begin{array}{l}683.33 \\
1036\end{array}$ & 0.001 \\
\hline LVI & $\begin{array}{l}\text { Yes } \\
\text { No }\end{array}$ & $\begin{array}{l}109(59.2) \\
75(40.8)\end{array}$ & $\begin{array}{l}810.06 \\
715.81\end{array}$ & 0.961 \\
\hline Metastasis+ & $\begin{array}{l}\text { Yes } \\
\text { No }\end{array}$ & $\begin{array}{l}32(17.4) \\
152(82.6)\end{array}$ & $\begin{array}{l}1204.04 \\
823.00\end{array}$ & 0.001 \\
\hline \multicolumn{5}{|c|}{$\begin{array}{l}\text { LVI: Lymphovascular invasion, SII: Systemic immune-inflammation index } \\
\text { +Metastasis: Lung metastasis, } 20 \text { patients; liver and lung metastasis: } 6 \text { patients } \\
\text { bone metastasis: } 5 \text { patients; brain metastasis: } 1 \text { patient }\end{array}$} \\
\hline
\end{tabular}

A cut-off point of 719 was obtained, with an area under the ROC (ROC) curve of 0.784 (Figure 1). The sensitivity at this cutoff point was $81 \%$, and the specificity was $65.4 \%$.

After the initial chemotherapy sessions, retroperitoneal lymph node dissection was performed in 10 patients. The median follow-up time was 55 (IQR 8-132) months. Ten patients died of TCa.

In the univariate Cox regression analysis, factors affecting OS were the presence of metastasis [hazard ratio (HR) $27.865 ; 95 \%$ Cl 3.274-3638.245; $p=0.03$ ], clinical stages II-III (HR 41.832; Cl 4.922-5461.637; $\mathrm{p}=0.02)$, pathological TNM classification over T2 (HR 24.054; Cl 2.818-3142.170; $p=0.04$ ), NLR (HR 1.789; Cl 1.170-2.669; $\mathrm{p}=0.006$ ), and SII (HR 1.004; Cl 1.243-1.875; $\mathrm{p}<0.001)$. In the multivariable analysis, SII (7.6-fold increase; HR $1.005 ; \mathrm{Cl} 1.279-4.251 ; \mathrm{p}=0.005)$ and presence of metastasis (4.3-fold increase; HR 1.710; Cl 0.279-6.730; $\mathrm{p}=0.001$ ) were independent predictors of OS. Although pathological TNM classification (HR 0.727; $\mathrm{Cl} 0.011-7.973 ; \mathrm{p}=0.80$ ) and NLR ratio (HR 0.398; $\mathrm{Cl} 0.027-1.162 ; \mathrm{p}=0.16$ ) affect the $\mathrm{OS}$, in the multivariable analysis, they did not affect OS. In addition, tumor size (HR 0.717; $\mathrm{Cl} 0.000-50108.01 ; \mathrm{p}=0.17$ ) and tumor type (seminoma or non-seminoma) (HR 0.398; Cl 0.069-1.796; $\mathrm{p}=0.28$ ) did not affect OS (Table 3).

In the Kaplan-Meier estimates of the probabilities of OS according to the SII, the cut-off value of SII was 719 to predict survival [-2Log (LR) 8.3178; $\mathrm{p}=0.003$ ) (Figure 2).

\section{Discussion}

In this study, the SII value was observed to be effective in predicting testicular tumor diagnosis. SII, especially between stage 1 and stages $2-3$, is more effective than other inflammatory

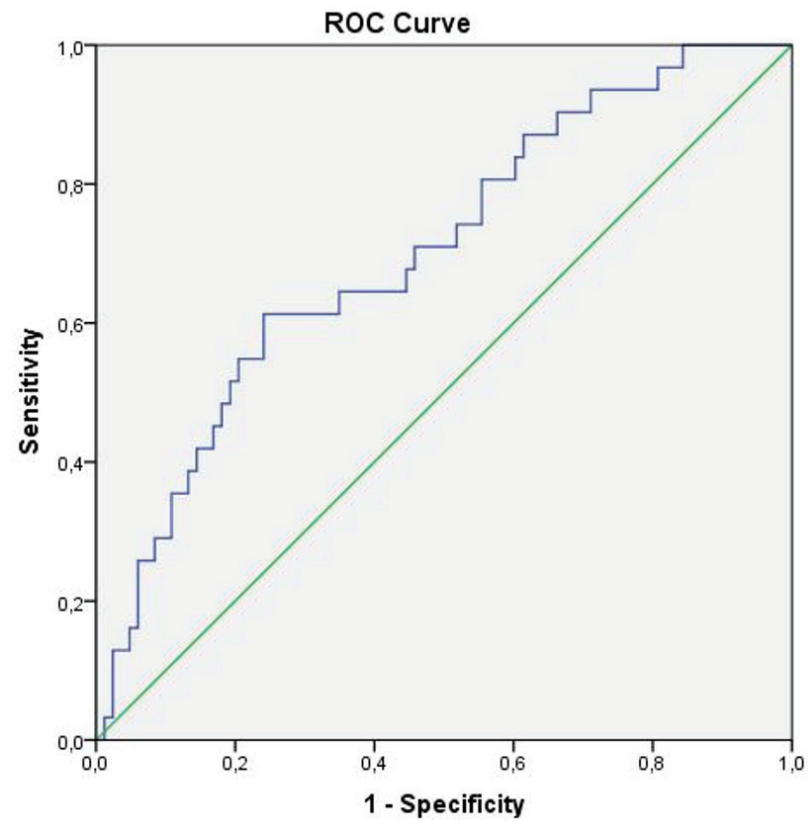

Figure 1. Receiver operating characteristics curve of systemic immuneinflammation index 
Göger et al. Prognostic Value of SII

\begin{tabular}{|c|c|c|c|c|c|c|}
\hline \multirow[b]{2}{*}{ Variables } & \multicolumn{3}{|c|}{ Univariate analysis } & \multicolumn{3}{|c|}{ Multivariate analysis } \\
\hline & Hazard ratio & Confidence interval & p-value & $\begin{array}{l}\text { Hazard } \\
\text { ratio }\end{array}$ & $\begin{array}{l}\text { Confidence } \\
\text { interval }\end{array}$ & p-value \\
\hline Metastasis & 27.865 & $3.274-3638.245$ & 0.03 & 1.710 & $0.155-6.730$ & 0.001 \\
\hline Pathological stage $\mathrm{T} 1-2$ vs $\geq \mathrm{T} 2$ & 24.054 & $2.818-3142.170$ & 0.04 & 0.727 & 0.011-7.973 & 0.80 \\
\hline Clinical stage I vs stage II-III & 13.831 & $0.021-707.844$ & 0.04 & 1.105 & $0.013-5.121$ & 0.01 \\
\hline Tumor type & 0.398 & $0.069-1.796$ & 0.27 & 0.813 & $0.002-4.128$ & 0.47 \\
\hline NLR & 1.789 & $1.170-2.669$ & 0.006 & 0.398 & $0.027-1.162$ & 0.16 \\
\hline SII & 1.004 & $1.243-1.875$ & $<0.001$ & 1.005 & $1.279-4.251$ & 0.005 \\
\hline
\end{tabular}

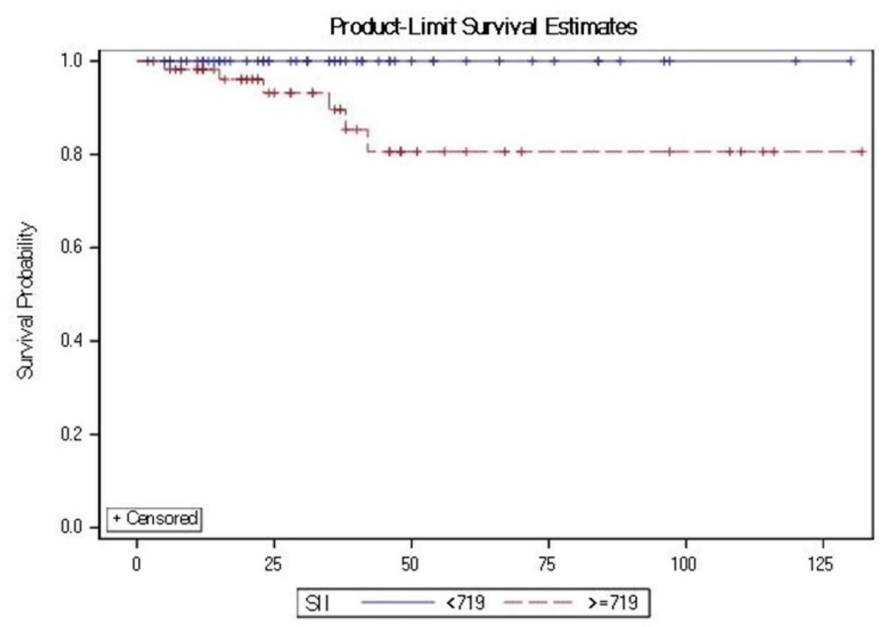

Figure 2. Kaplan-Meier estimates of the probabilities of the overall survival according to the systemic immune-inflammation index

markers and can be used in clinical evaluation and followup. The SII value was determined to be one of the important factors affecting testicular tumor prognosis. The inflammatory reaction plays a significant role in the development and prognosis of tumors in several ways, from the genesis of the tumor, progression, to metastasis (17). Inflammation-associated peripheral cells (neutrophils, lymphocytes, and Plt) obtained from the peripheral blood are associated with the progression of various tumors $(13,14)$. In addition, inflammatory indices (II) derived from different combinations of these peripheral cells, such as the NLR and platelet-to-lymphocyte ratio (PLR) have been evaluated in various prognostic factor studies of malignant solid tumors $(9,18,19)$.

SII, a recent inflammatory index, is calculated as follows: SII $=P^{*} N / L$, where $P, N$, and $L$ are the peripheral Plts, neutrophil count, and lymphocyte count (17). It has recently been investigated as a prognostic marker in various malignancies. Studies have suggested that SII is superior to alternative systemic inflammation indices such as PLR and NLR and could serve as a more objective marker that reflects the balance between host inflammatory and immune response status $(8,20,21)$.

One of the first studies of TCa using complete blood count indices was conducted using NLR. Şahin et al. (22) reported that
NLR was higher in the TCa group than in the varicocele group. Yuksel et al. (23) eported that the NLR is a simple and effective marker in TCa stage I (23). However, Jankovich et al. (24) could not find a difference between metastatic and non-metastatic $\mathrm{TCa}$ in their study, but they determined significance in cancer grades above T1 in NLR $<4$ according to the TNM classification. Tan et al. (25) revealed NLR $\geq 3.0$ and above as significant in patients with lymph node involvement and patients with metastasis, which had worse CSS. In the present study, the NLR was significantly higher in patients with TCa. Moreover, OS was determined in the univariate analysis.

To the best of our knowledge, this study is the first to compare the relationship between SII and TCa with a control group. SII increases significantly in TCa, with an area under the ROC curve of 0.784 (Figure 1). The sensitivity and specificity at this cut-off point were $81 \%$ and $65.4 \%$, respectively. The findings of this study suggest that SII can be used effectively in the diagnosis of TCa.

The SII value is closely related to those reported by studies evaluating the prognosis of urological cancers (26). The median progression-free survival was 6.3 months in patients with metastatic renal cell carcinoma with SII $\geq 730$ and 18.7 months in those with SII <730 (27). In another study, Lolli et al. (28) examined patients with metastatic prostate cancer and reported an overall median OS of 17.3 months, with 21.8 months in the SII $<535$ group and 14.7 months in the SII $\geq 535$ $(p<0.0001)$. A retrospective study of muscle-invasive bladder cancer determined that $\mathrm{SII}>843$ is considered a poor prognostic criterion (29). Chovanec et al. (16) conducted one of the rare studies on TCa and SII, and they determined a median SII value of 1003 in patients with metastatic TCa. In the multivariate Cox analysis, the OS in the poor prognostic group according to IGCCCG was affected by SII. However, progression-free survival was not affected.

Fankhauser et al. (15) reported that high SII, neutrophil, and NRL values, together with IGCCCG risk groups, are prognostic predictors of OS in metastatic TCa before first-line chemotherapy. In their ROC analysis of SII, they revealed that 1428 was the ideal cut-off value for clinical decision making. In the subgroup analysis, although neutrophils and leukocytes were high in patients with bone visceral organ and brain metastases, no difference was found in NRL and SII. 
Especially, Fankhauser et al. (15) and Chovanec et al. (16) found that SII had potential to provide a more efficient prediction of oncological outcomes in patients with metastatic GCT compared with the well-established IGCCCG classification system. In the present study, in which the mean SIl value was 719, the SII value increased to 1036 in cases with advanced stages. The fact that the majority of our participants had stage 1 TCa might be the underlying reason for the lower SII value obtained when compared with other reported values. However, it was the highest in the metastatic group. In addition, unlike other studies, the present study compared the relation of SII level with pathology type, LVI, and tumor size. In line with similar studies, the present study found a cooperative relationship was found between clinical stage and metastasis and SII. It is one of the factors that affect OS. Improved prediction of oncological outcomes could affect the oncologists' decisions concerning systemic treatment and thus might enable a more personalized and eventually a more effective treatment option for eligible patients with metastatic GCT.

In this study, the majority of the patients had TCa stages 1 and 2. Patients with seminoma and non-seminoma GCT had a 6\%$18 \%$ risk of recurrence even those in the low-risk groups. At the time of diagnosis, $15 \%$ and $50 \%$ of the patients with seminomas and non-seminomas, respectively, have subclinical stage 2 as determined during patient surveillance. One of the important points is that we can classify patients correctly at these stages, initiate effective treatment, and predict the risk of recurrence after treatment.

Considering other current studies, this study is the first to evaluate SII values with the clinical stage of TCa. Especially, identifying patients with high-risk $\mathrm{TCa}$ at the time of initial diagnosis requires a closer follow-up program or an intensified treatment algorithm. In the present study, SII values can be used in the diagnosis and defining the treatment modality of patients with high-risk status.

In patients with TCa stage 1, the treatment plan is made according to pathological prognostic factors. Metastatic TCa treatment preferences are based on IGCCCG classification. Despite surgery and adjuvant chemotherapy, progression, and metastasis can be seen in some patients with stage $1 \mathrm{TCa}$, while some may experience serious side effects of treatment. However, despite IGCCCG risk estimation and effective chemotherapy regimens, first-line chemotherapy fails in some patients with metastatic TCa and may die eventually. These classifications have been in use for over 20 years. At present, available data are mostly based on findings from the 1990s. Since then, advances in diagnostics (mainly imaging), as well as new treatment protocols and more standardized follow-up regimens, have also been applied to the management of TCa.

The TCa update using a more up-to-date cohort is currently under preparation (30). With updated information, it is possible to better predict oncological outcomes and plan the treatment algorithm in early-stage or metastatic GCT. Better risk stratification is possible by combining conventional clinical and pathological data with new biomarkers, genetic tests, and new imaging techniques.

\section{Study Limitations}

This study has some limitations. First, the study has a retrospective design and may be underrepresented in earlystage and metastatic data. Second, some imbalances exist between datasets that could be responsible for the differences in the median SII value. Third, most of the participants had stage 1 and 2 diseases, which might have affected the data obtained. SII could yield more accurate data in patients with advanced disease stages.

\section{Conclusion}

SIl is a simple examination that can be evaluated through a simple blood test. SII has the potential to contribute to disease-specific diagnosis and treatment algorithms. It can provide additional information to urologists, especially in post-treatment follow-up. However, more cohort, prospective studies are needed.

\section{Acknowledgements}

Publication: The results of the study were not published in full or in part in form of abstracts.

Contribution: There is not any contributors who may not be listed as authors.

Conflict of Interest: No conflict of interest was declared by the authors.

Financial Disclosure: The authors declared that this study received no financial support.

\section{Ethics}

Ethics Committee Approval: The study was approved by the Meram Medical Faculty Ethics Committee of Necmettin Erbakan University (protocol no: 2021/2980).

Informed Consent: Written informed consent was obtained from all patients.

Peer-review: Externally peer-reviewed.

\section{Authorship Contributions}

Supervision: Y.E.G., M.A., Concept: Y.E.G., M.S.Ö., Design: Y.E.G., M.S.Ö., Data Collection or Processing: M.S.Ö., H.U., Y.E.G., M.K., Analysis or Interpretation: H.U., M.K., Literature Search: Y.E.G., M.S.Ö., M.K., H.U., M.A., Writing: Y.E.G.

\section{References}

1. Park JS, Kim J, Elghiaty A, Ham WS. Recent global trends in testicular cancer incidence and mortality. Medicine (Baltimore) 2018;97:e12390. doi: 10.1097/MD.0000000000012390.

2. Pishgar F, Haj-Mirzaian A, Ebrahimi $\mathrm{H}$, et al. Global, regional and national burden of testicular cancer, 1990-2016: results from the Global Burden of Disease Study 2016. BJU Int 2019;124:386-394.

3. Gurney JK, Florio AA, Znaor A, et al. International trends in the incidence of testicular cancer: lessons from 35 years and 41 countries. Eur Urol 2019;76:615-623.

4. Gilligan T, Lin DW, Aggarwal R, et al. Testicular cancer, version 2.2020, NCCN clinical practice guidelines in oncology. J Natl Compr Canc Netw 2019;17:1529-1554.

5. Siegel RL, Miller KD, Jemal A. Cancer statistics, 2019. CA Cancer J Clin 2019;69:7-34. 
6. Verhoeven RH, Karim-Kos HE, Coebergh JWW, et al. Markedly increased incidence and improved survival of testicular cancer in the Netherlands. Acta Oncol 2014;53:342-350.

7. Hanahan D, Weinberg RA. Hallmarks of cancer: the next generation. Cell 2011;144:646-674.

8. Wang K, Diao F, Ye Z, et al. Prognostic value of systemic immuneinflammation index in patients with gastric cancer. Chin J Cancer 2017;36:75.

9. Templeton AJ, McNamara MG, Šeruga B, et al. Prognostic role of neutrophil-to-lymphocyte ratio in solid tumors: a systematic review and meta-analysis. J Natl Cancer Inst 2014;106:dju124. doi: 10.1093/ jnci/dju124.

10. Santoni M, De Giorgi U, lacovelli R, et al. Pre-treatment neutrophilto-lymphocyte ratio may be associated with the outcome in patients treated with everolimus for metastatic renal cell carcinoma. $\mathrm{Br}$ J Cancer 2013;109:1755.

11. Rossi L, Santoni M, Crabb SJ, et al. High neutrophil-to-lymphocyte ratio persistent during first-line chemotherapy predicts poor clinical outcome in patients with advanced urothelial cancer. Ann Surg Oncol 2015;22:1377-1384.

12. Hermanns T, Bhindi B, Wei $Y$, et al. Pre-treatment neutrophil-tolymphocyte ratio as predictor of adverse outcomes in patients undergoing radical cystectomy for urothelial carcinoma of the bladder. Br J Cancer 2014;111:444-451.

13. Seruga B, Zhang H, Bernstein LJ, Tannock IF. Cytokines and their relationship to the symptoms and outcome of cancer. Nat Rev Cancer 2008;8:887-899.

14. Cools-Lartigue J, Spicer J, McDonald B, et al. Neutrophil extracellular traps sequester circulating tumor cells and promote metastasis. J Clin Invest 2013;123:3446-3458.

15. Fankhauser $C D$, Sander $S$, Roth $L$, et al. Systemic inflammatory markers have independent prognostic value in patients with metastatic testicular germ cell tumours undergoing first-line chemotherapy. $\mathrm{Br}$ J Cancer 2018;118:825-830.

16. Chovanec M, Cierna Z, Miskovska V, et al. Systemic immuneinflammation index in germ-cell tumours. $\mathrm{Br}$ J Cancer 2018;118:831-838.

17. Hu B, Yang $X-R, X u$ Y, et al. Systemic immune-inflammation index predicts prognosis of patients after curative resection for hepatocellular carcinoma. Clin Cancer Res 2014;20:6212-6222.

18. Proctor M, McMillan D, Morrison D, et al. A derived neutrophil to lymphocyte ratio predicts survival in patients with cancer. Br J Cancer 2012;107:695-699.
19. Templeton AJ, Ace O, McNamara MG, et al. Prognostic role of platelet to lymphocyte ratio in solid tumors: a systematic review and metaanalysis. Cancer Epidemiol Biomarkers Prev 2014;23:1204-1212.

20. Geng Y, Shao Y, Zhu D, et al. Systemic immune-inflammation index predicts prognosis of patients with esophageal squamous cell carcinoma: a propensity score-matched analysis. Sci Rep 2016;6:39482.

21. Zhong J-H, Huang D-H, Chen Z-Y. Prognostic role of systemic immune-inflammation index in solid tumors: a systematic review and meta-analysis. Oncotarget 2017;8:75381.

22. Şahin A, Toprak T, Kutluhan MA, et al. Increased neutrophil/ lymphocyte ratio in testicular cancer. Arch Ital Urol Androl 2019;91. doi: 10.4081/aiua.2019.2.97.

23. Yuksel $\mathrm{OH}$, Verit $\mathrm{A}$, Sahin $\mathrm{A}$, et al. White blood cell counts and neutrophil to lymphocyte ratio in the diagnosis of testicular cancer: a simple secondary serum tumor marker. Int Braz J Urol 2016;42:53-59.

24. Jankovich M, Jankovichova T, Ondrus D, Breza J. Neutrophil-tolymphocyte ratio as a predictor of preoperative tumor staging in testicular germ cell tumors. Bratisl Lek Listy 2017; 118:510-512.

25. Tan YG, Sia J, Huang HH, Lau WKO. Neutrophil-to-lymphocyte ratio independently predicts advanced pathological staging and poorer survival outcomes in testicular cancer. Invest Clin Urol 2019;60:176-183.

26. Huang $Y$, Gao $Y$, Wu Y, Lin $\mathrm{H}$. Prognostic value of systemic immuneinflammation index in patients with urologic cancers: a meta-analysis. Cancer Cell Int 2020;20:499.

27. Lolli C, Basso U, Derosa L, et al. Systemic immune-inflammation index predicts the clinical outcome in patients with metastatic renal cell cancer treated with sunitinib. Oncotarget 2016;7:54564-54571.

28. Lolli C, Caffo O, Scarpi E, et al. Systemic immune-inflammation index predicts the clinical outcome in patients with mCRPC treated with abiraterone. Front Pharmacol 2016;7:376.

29. Gorgel SN, Akin Y, Koc EM, et al. Retrospective study of systemic immune-inflammation index in muscle invasive bladder cancer: initial results of single centre. Int Urol Nephrol 2020;52:469-473.

30. Collette L. Update of the international prognostic classification for first line metastatic germ-cell cancers. An international initiative. Eur J Cancer 2017; 1:S196-S197. 